\title{
Endoscopic versus percutaneous management for symptomatic pancreatic fluid collections: a systematic review and meta-analysis
}

\section{(ㄷ)(1) $(9)$}

\author{
Authors \\ Muhammad Ali Khan ${ }^{1,}{ }^{,}$, Tariq Hammad ${ }^{2,}{ }^{,}$, Zubair Khan ${ }^{3}$, Wade Lee ${ }^{4}$, Monica Gaidhane ${ }^{5}$, Amy Tyberg ${ }^{5}$, Michel \\ Kahaleh $^{5}$
}

Institutions

1 Division of Gastroenterology, University of Tennessee Health Science Center, Memphis, TN, USA

2 Division of Gastroenterology, Baylor College of Medicine, Houston, TX, USA

3 Division of Gastroenterology, University of Toledo, Toledo, OH, USA

4 Mulford and Carlson Libraries, University of Toledo, Toledo, OH, USA

5 Department of Medicine - Rutgers Robert Wood Johnson Medical School Rutgers, The State University of New Jersey, Robert Wood Johnson University Hospital

submitted 5.12 .2017

accepted after revision 25.1.2018

\author{
Bibliography \\ DOI https://doi.org/10.1055/s-0044-102299 | \\ Endoscopy International Open 2018; 06: E474-E483 \\ (c) Georg Thieme Verlag KG Stuttgart · New York \\ ISSN 2364-3722
}

Corresponding author

Michel Kahaleh, MD, AGAF, FACG, FASGE, Professor of Medicine, Clinical Director of Gastroenterology, Chief of Endoscopy, Director Pancreas Program, Department of Medicine - Rutgers Robert Wood Johnson Medical School, Rutgers, The State University of New Jersey, Robert Wood Johnson University Hospital, 1 RWJ Place, MEB 464, New Brunswick, NJ 08901, USA

Fax: 732-235-5537

mkahaleh@gmail.com

\section{ABSTRACT}

Background Symptomatic pancreatic fluid collections (PFCs) are managed by surgical, percutaneous, or endoscopic drainage. Due to morbidity associated with surgical drainage, percutaneous and/or endoscopic options have increasingly been used as initial management.

Aims We conducted a systematic review and meta-analysis comparing the efficacy and safety of endoscopic versus percutaneous drainage for management of PFCs.

Methods We searched several databases from inception through 31 August 2017 to identify comparative studies using endoscopic or percutaneous drainage for PFCs. Our primary outcome was clinical success. Secondary outcomes were technical success, adverse events (AE), rates of recurrence, requirement for subsequent procedures, and length of stay in hospital. Pooled risk ratios (RR) and mean difference (MD) were calculated for categorical and continuous outcomes, respectively.

Results Seven studies with 490 patients were included in the final analysis. Pooled RR for clinical success was 0.40 $(0.26,0.61), P^{2}=42 \%$ in favor of endoscopic management. On sensitivity analysis, after excluding one study on patients with walled-off necrosis (WON), the clinical success was $0.43(0.28,0.66)$ with no heterogeneity. Pooled RR for technical success was $1.50(0.52,4.37)$ with no heterogeneity. Pooled RR for AE and rate of recurrence were 0.77 $(0.46,1.28)$ and $0.60(0.29,1.24)$, respectively. Pooled MD for length of stay in hospital and rate of re-intervention were $-8.97(-12.88,-5.07)$ and $-0.66(-0.93,-0.38)$, respectively, in favor of endoscopic drainage.

Conclusions Endoscopic drainage should be the preferred therapeutic modality for PFCs compared to percutaneous drainage as it is associated with significantly better clinical success, a lower re-intervention rate, and a shorter hospital length of stay.

\section{Introduction}

Acute pancreatitis is the most common gastroenterological cause for inpatient admission in the United States and subse-

* Authors contributed equally to the manuscript. quently results in substantial morbidity, mortality, and cost 
burdens [1]. Pancreatic fluid collections (PFCs) are common complications of pancreatitis and may occur in up to $20 \%$ of necrotizing pancreatitis [2,3]. Based on the acuity and content, PFCs are classified according to the revised Atlanta classification into four distinctive categories: acute peripancreatic fluid collection (APFC), acute necrotic collections (ANC), pancreatic pseudocysts (PPs) and walled-off necrosis (WON) [4]. Additionally, about $15 \%$ to $30 \%$ of patients undergoing distal pancreatic resections develop postoperative PFCs due to pancreatic duct leaks [5-7].

Drainage of PFCs is indicated if they become infected or symptomatic. This can be performed by surgical, percutaneous, or endoscopic approaches. Surgical drainage, both open and minimally invasive retroperitoneal necrosectomy, has been associated with high rates of morbidity and mortality with a $47 \%$ to $72 \%$ complication rate [8 - 10]. Endoscopic drainage (ED) and percutaneous drainage (PD) have widely replaced surgical drainage as initial, efficacious, and less invasive approaches for symptomatic PFCs. Despite having comparable clinical efficacy, in some studies PD was associated with a higher rate of fistula formation, a higher rate of re-interventions, a longer length of hospital stay, and a higher median number of follow-up abdominal imaging studies compared to ED [11, 12]. Over the past two decades, advances in endoscopic techniques have made ED the preferred drainage modality. The use of endoscopic ultrasound (EUS) allows for assessment of the collection, creation of an internal fistulous tract even into collections not bulging into the gastrointestinal lumen, avoidance of intervening vasculature, and deployment of internal drainage stents. Direct endoscopic necrosectomy (DEN) is a rapidly evolving technique in which endoscopic debridement of the PFC (WON in particular) is performed through the cyst-gastrostomy or cyst-duodenostomy stent [13]. Several types of stent have been used for ED: plastic double pigtail stents, fully covered self-expanding metal stents (FCSEMS) and more recently, lumen apposing metal stents (LAMS). LAMS have made DEN easier, more efficient, and with no difference in cost compared to plastic stent usage $[14,15]$.

Few studies have compared PD with ED for management of symptomatic PFC. The aim of the present systematic review and meta-analysis is to compare the efficacy (technical success and clinical success) and safety (adverse events and rate of recurrence) of endoscopic versus percutaneous drainage for management of PFCs based on the information available as of August 2017.

\section{Methods}

\section{Identification and retrieval of primary studies}

The systematic review and meta-analysis was conducted according to the guidelines of preferred reporting items for systematic reviews and meta-analyses (PRISMA) [16]. The search strategy and subsequent literature search were developed in consultation with an experienced medical reference librarian (WML). The search strategies were developed in Medline and then replicated using the same subject headings and keywords for Cochrane database, EMBASE, and Web of Science from in- ception through 31 August 2017. Search terms included: "pancreatic fluid collections", "pancreatic pseudocyst", "walled off necrosis", "percutaneous drainage", and "endoscopic drainage". The search accounted for plurals and variations in spelling with the use of appropriate wildcards. There was no restriction of language. The full search strategy is available as Appendix 1. Articles were selected for full text review on the basis of their title and abstract. To increase the yield of our search strategy, we manually searched references and related citations then cross-referencing was performed for articles identified. All results were downloaded into EndNote 7.5 (Thompson ISI ResearchSoft, Philadelphia, Pennsylvania, United States), a bibliographic database manager; any duplicate citation was identified and removed.

\section{Study selection, data extraction, and quality assessment}

Eligibility criteria were determined a priori by two study authors (MAK and MK) and included comparative studies using endoscopic or percutaneous drainage for symptomatic PFC. Endoscopic drainage could be achieved by transmural and/or transpapillary route, with or without the use of endoscopic ultrasound (EUS).

Studies were included only if they reported clinical success (clinical and radiological resolution) and post-procedure adverse events. Abstracts were excluded only if data presented initially were later published as a full peer reviewed journal article, in which case the fully published study was included. Corresponding authors of studies were contacted when required data from studies was not available or there was concern for overlap of patients among studies. Abstracts of Digestive Disease Week were searched manually from year 2012 up to 2017 for any comparative studies. Two independent reviewers ( $\mathrm{TH}$ and MAK) reviewed the title and abstract search with inclusion decisions for each study made independently based on the eligibility criteria. Any disagreement between reviewers was discussed with a third reviewer (MK) and agreement was reached by consensus.

Data from included studies were independently extracted by two reviewers (ZK and MAK). Extracted data included study design, country, year of publication, patient demographics, type of PFC, etiology of the PFC, site and size of the PFC, DEN, drainage approach, clinical success, technical success, rate of recurrence, length of stay in hospital, and adverse events. After complete extraction of data, the data sheets were compared and any disagreement between reviewers was to be discussed with a third reviewer (MK) with agreement reached by consensus.

Quality of studies was assessed independently by two investigators (MAK and ZK) using the Newcastle Ottawa scale (NOS) for observational studies [17]. Any discrepancy in quality assessment between reviewers was discussed with a third reviewer (MK) with agreement reached by consensus.

\section{Data synthesis and statistical analysis}

All statistical analyses were conducted using Review Manager (RevMan, version 5.3 for Windows; The Cochrane Collaboration, The Nordic Cochrane Centre, Copenhagen, Denmark) and 
3892 records identified from database search

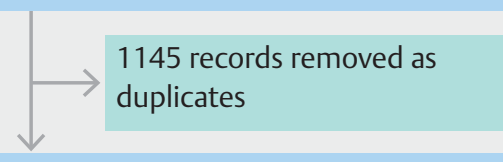

2747 records screened after duplicates removal

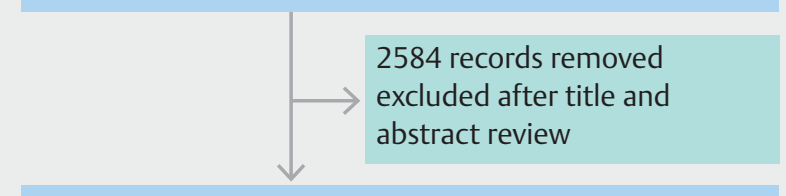

163 full-text articles from database search reviewed

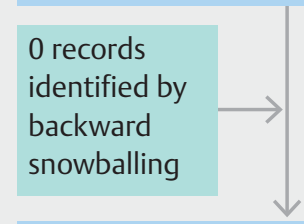

163 full-text articles assessed for eligibility

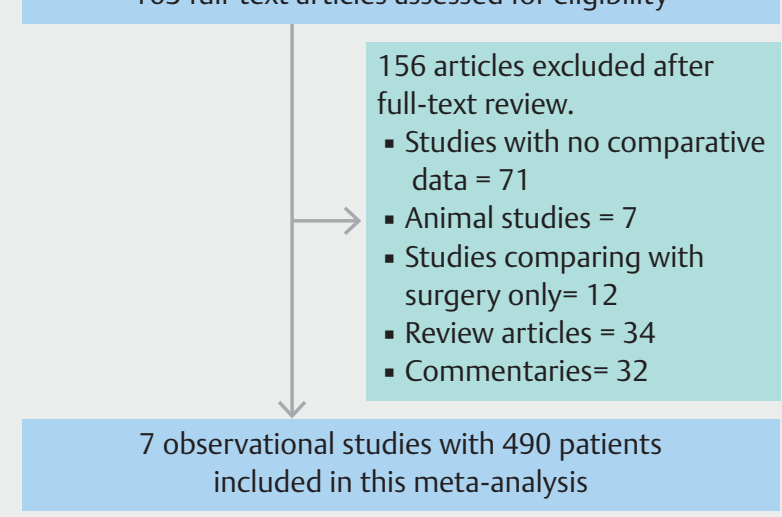

Fig. 1 PRISMA flow chart summarizing the study selection process.

Comprehensive Meta-analysis (version 3.0; Englewood, NJ, United States). The primary outcome was clinical success (clinical and radiological resolution). Secondary outcomes were technical success, adverse events (AE), rates of recurrence, requirement for subsequent procedures, and length of stay in hospital. Pooled risk ratios (RR) and mean difference (MD) were calculated for categorical and continuous outcomes, respectively, and analyzed using a fixed effect model, unless heterogeneity was encountered, in which case we used a random effects model. When studies included zero events in both arms, continuity correction was applied to include the zero-event study in the pooled estimate $[18,19]$. To assess heterogeneity, we used $R$ statistics and Cochran's $Q$ test, in which a $P$ value $<0.1$ for $\mathrm{Co}$ chran's $\mathrm{Q}$ test indicated the presence of heterogeneity. The ${ }^{2}$ values of $>50 \%$ were consistent with significant heterogeneity [20]. We expected heterogeneity in our estimate as various etiologies of PFCs were included. Therefore, a sensitivity analysis was conducted after excluding study comparing performance in WON. We also conducted a subgroup analysis based on type of endoscopic drainage, i.e. EUS-guided transmural drain- age exclusively and endoscopic drainage including both transmural and/or transpapillary methods. We assessed publication bias with funnel plots and Egger's test if deemed necessary.

\section{Results}

The search strategy identified 3892 citations of which 1145 were removed as duplicates. Out of the remaining 2747 articles, 2584 were removed after title and abstract review. Bibliographic search of 163 articles did not reveal any additional study. Therefore, a total of 163 articles were reviewed of which 7 observational studies [10,11,21-25] with 490 patients were included in this systematic review and meta-analysis. An abstract [26], initially presented at Digestive Disease Week 2012, was excluded as results from this study were incorporated into a single center study published in 2014 [11]. The search strategy is highlighted in $>$ Fig. 1.

\section{Study characteristics and quality assessment}

All seven studies $[10,11,21-25]$ were retrospective observational studies. Among 490 patients, 242 underwent endoscopic drainage while the remaining 248 underwent percutaneous drain placement. Three studies $[21,22,25]$ exclusively evaluated PFCs after pancreatic surgery $(n=189)$, two studies $[11,24]$ were comprised of PP patients only $(n=112)$, one study [10] focused on WON patients only $(n=24)$, and the remaining one study [23] included both WON and PP. In patients who underwent endoscopic management, two studies [21,23] used both plastic and metal stents at the discretion of the endoscopist, four studies $[10,11,22,24]$ used plastic stents exclusively, and one study did not mention the type of stents used [25]. Patient demographics are highlighted in $>$ Table 1. Two studies $[10,22]$ exclusively used EUS for endoscopic management with the use of one to three $(7-10 \mathrm{Fr})$ pigtail plastic stents. In the remaining studies, EUS was used at the discretion of the endoscopist.

Quality assessment of studies was conducted using NOS assessment tool. All seven studies were rated as moderate quality studies. This tool assesses quality in three parameters of selection, comparability, and exposure/outcome, and allocates a maximum of 4, 2, and 3 points, respectively. High quality studies are scored greater than 7 on this scale, and moderate quality studies, between 5 and 7 . Detailed quality assessment is presented in $>$ Table 2 .

\section{Meta-analysis}

Our primary outcome of interest was clinical success which was defined as radiological resolution of PFC, along with improvement in clinical symptoms leading to subsequent removal of drains and stents. All seven studies [10,11,21-25] presented data on clinical success. Pooled RR with $95 \%$ confidence interval $(\mathrm{Cl})$ was $0.40(0.26,0.61)$, Cochran's $Q$ test $P=0.11, R^{2}=$ $42 \%$ ( $\mathbf{F i g . 2}$ ). Funnel plot appeared asymmetric but Egger's test failed to detect any publication bias (intercept $=0.14, P=$ 0.91 , two-tailed). On sensitivity analysis, after excluding the study with WON, pooled RR with $95 \% \mathrm{Cl}$ was $0.43(0.28,0.66)$, Cochran's $Q$ test $P=0.13, R^{2}=41 \%$. On subgroup analysis, 


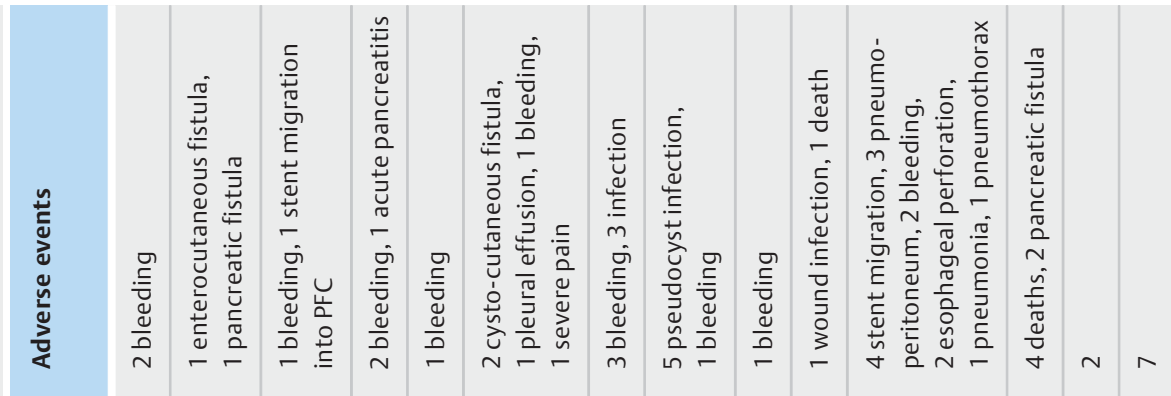

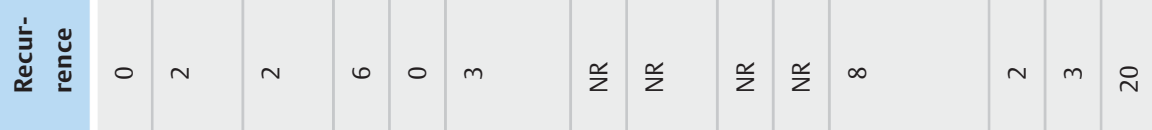

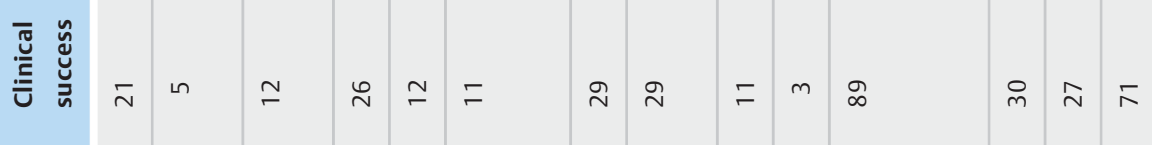

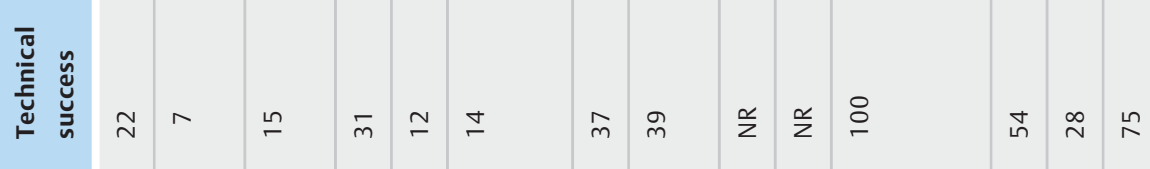

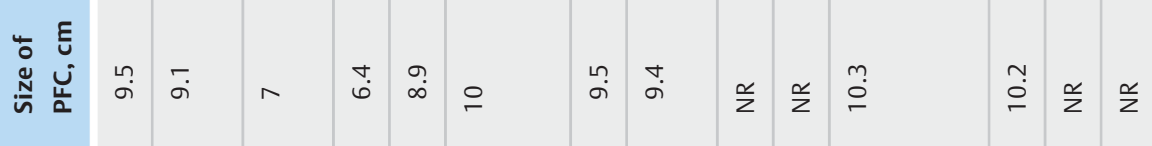

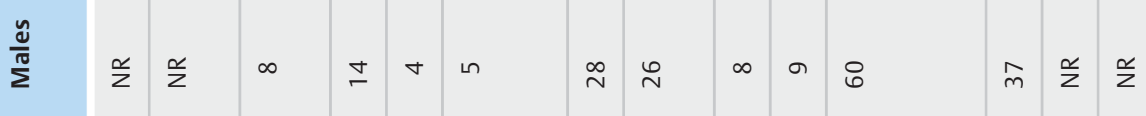

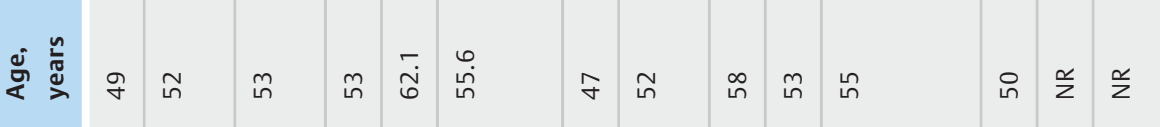

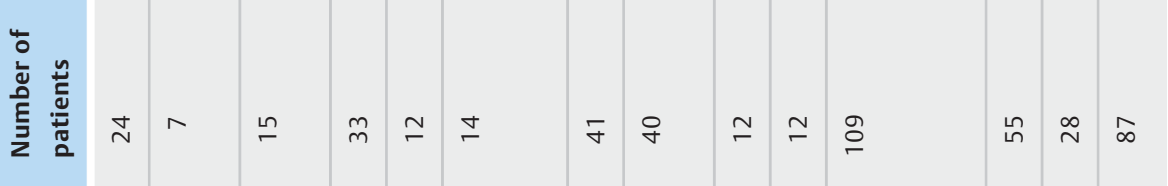

定

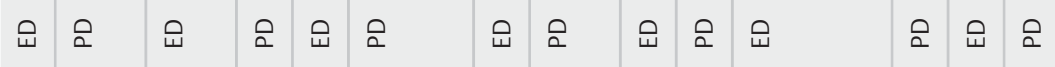

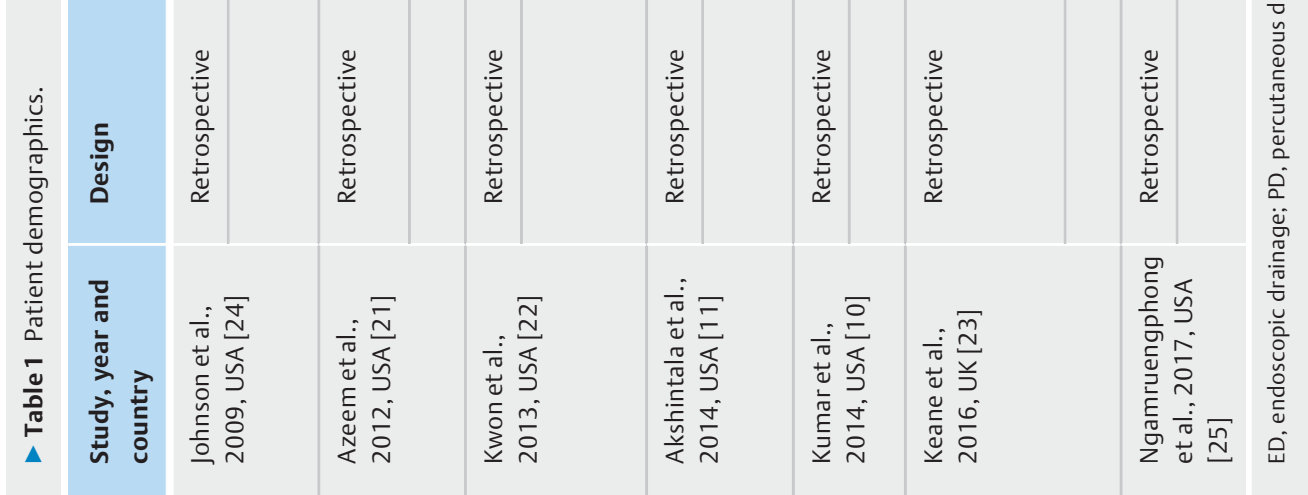


- Table 2 Quality assessment of studies using Newcastle Ottawa Scale.

\begin{tabular}{|c|c|c|c|c|c|c|c|c|c|}
\hline \multirow[t]{2}{*}{ Study } & \multicolumn{4}{|l|}{ Selection } & \multirow{2}{*}{$\begin{array}{l}\text { Comparability } \\
\text { Adjustment } \\
\text { for primary } \\
\text { and secondary } \\
\text { factors }\end{array}$} & \multicolumn{3}{|l|}{ Outcome } & \multirow[t]{2}{*}{ Quality } \\
\hline & $\begin{array}{l}\text { Representa- } \\
\text { tiveness of } \\
\text { exposed } \\
\text { cohort }\end{array}$ & $\begin{array}{l}\text { Selection } \\
\text { of non- } \\
\text { exposed } \\
\text { cohort }\end{array}$ & $\begin{array}{l}\text { Ascer- } \\
\text { tainment } \\
\text { of expo- } \\
\text { sure }\end{array}$ & $\begin{array}{l}\text { Outcome } \\
\text { not pres- } \\
\text { ent at } \\
\text { start }\end{array}$ & & $\begin{array}{l}\text { Assess- } \\
\text { ment by } \\
\text { record } \\
\text { linkage }\end{array}$ & $\begin{array}{l}\text { Long e- } \\
\text { nough fol- } \\
\text { low-up for } \\
\text { outcome } \\
\text { to occur }\end{array}$ & $\begin{array}{l}\text { Ade- } \\
\text { quacy } \\
\text { of fol- } \\
\text { low-up }\end{array}$ & \\
\hline $\begin{array}{l}\text { Johnson et al., } \\
2009 \text { [24] }\end{array}$ & + & + & + & + & - & + & + & + & $\begin{array}{l}\text { Moderate } \\
\text { quality }\end{array}$ \\
\hline $\begin{array}{l}\text { Azeem et al., } \\
2012 \text { [21] }\end{array}$ & + & + & + & + & - & + & + & + & $\begin{array}{l}\text { Moderate } \\
\text { quality }\end{array}$ \\
\hline $\begin{array}{l}\text { Kwon et al., } \\
2013 \text { [22] }\end{array}$ & + & + & + & + & - & + & + & + & $\begin{array}{l}\text { Moderate } \\
\text { quality }\end{array}$ \\
\hline $\begin{array}{l}\text { Akshintala et } \\
\text { al., } 2014 \text { [11] }\end{array}$ & + & + & + & + & - & + & + & + & $\begin{array}{l}\text { Moderate } \\
\text { quality }\end{array}$ \\
\hline $\begin{array}{l}\text { Kumar et al., } \\
2014 \text { [10] }\end{array}$ & + & + & + & + & - & + & - & - & $\begin{array}{l}\text { Moderate } \\
\text { quality }\end{array}$ \\
\hline $\begin{array}{l}\text { Keane et al., } \\
2016 \text { [23] }\end{array}$ & + & + & + & + & - & + & + & + & $\begin{array}{l}\text { Moderate } \\
\text { quality }\end{array}$ \\
\hline $\begin{array}{l}\text { Ngamrueng- } \\
\text { phong et al., } \\
2017 \text { [25] }\end{array}$ & + & + & + & + & - & + & - & - & $\begin{array}{l}\text { Moderate } \\
\text { quality }\end{array}$ \\
\hline
\end{tabular}

pooled RR in EUS guided transmural drainage exclusively and endoscopic transmural and/or transpapillary drainage were $0.13(0.03,0.61), R^{2}=0 \%$ and $0.44(0.28,0.68), R^{2}=51 \%$, respectively ( $\triangleright$ Fig. 2 ). Technical success was defined as completion of index procedure with successful stent or drain placement in the intended position as determined endoscopically or radiologically. Six studies [11,21-25] with 466 patients compared technical success. Pooled RR was $1.50(0.52,4.37)$, Cochran's Q test $P=0.34, R=12 \%$ ( $/$ Fig. 3 ). No publication bias was detected with funnel plot and Egger's test of asymmetry (intercept 5.21, $P=0.67$, two-tailed). Although we did not find any difference in technical success, clinical success was significantly better in the endoscopic drainage group. This was consistent, even after exclusion of the study evaluating WON exclusively.

Adverse events were defined as post procedure complications which required any additional intervention or resulted in hospital stay. All seven studies reported data on adverse events. Pooled RR for overall adverse events was $0.77(0.46$, 1.28), Cochran's $Q$ test $P=0.75, P^{2}=0 \%$ ( $\triangleright$ Fig. 4 ). No publication bias was detected with funnel plot and Egger's test (intercept $=-1.56, P=0.09$, two-tailed). On subgroup analysis, pooled RR for exclusive EUS guided transmural drainage and endoscopic transmural and/or transpapillary drainage were $0.33(0.07,1.46), I^{2}=0 \%$ and $0.86(0.50,1.49), I^{2}=0 \%$, respectively. Bleeding was the most common adverse event, occurring in $4 \%$ of patients in the endoscopic group and $1.5 \%$ in the percutaneous group. However, this difference was not statistically significant $(P=0.10)$. Five deaths occurred during 30day follow-up in the percutaneous group, while no deaths occurred in the endoscopic group. Once again, this difference was not statistically significant $(P=0.16)$. Recurrence was defined as reoccurrence of the collection after removal of the drain or stent. Five studies [21-25] evaluated recurrence rate with both procedures. Pooled RR was 0.60 (0.29, 1.24), Cochran's $Q$ test $P=0.22, P^{2}=30 \%$ ( $>$ Fig. 5). Three studies [10, $23,25]$ provided data on requirement for re-interventions; pooled mean difference was $-0.66(-0.93,-0.38)$, Cochran's $\mathrm{Q}$ test $P=0.01, P=77 \%$ ( $\triangleright$ Fig.6a). Finally, length of stay in days was compared in three studies; pooled mean difference with $95 \% \mathrm{Cl}$ was $-8.97(-12.88,-5.07)$, Cochran's Q test $P=$ $0.13, R^{2}=51 \%$ ( Fig. 6b).

\section{Discussion}

Due to the invasive nature and morbidity associated with surgical drainage of symptomatic PFCs, percutaneous and/or endoscopic options have increasingly been used as initial management. Percutaneous drainage can be performed under local anesthesia avoiding the need for monitored or general anesthesia; however, percutaneous drainage requires an external catheter which compromises patients' quality of life, requiring daily care and maintenance and may result in localized skin irritation. Endoscopic drainage allows for larger diameter internal stent placement, avoiding the need for external drains, allowing for DEN, and allowing for reintroduction of pancreatic fluid back into the gastrointestinal tract which is more physiological compared to percutaneous drainage where pancreatic fluid is lost. However, it requires general anesthesia to be performed safely. While both are efficacious in published studies, one has not been definitively identified as superior to the other, justifying 


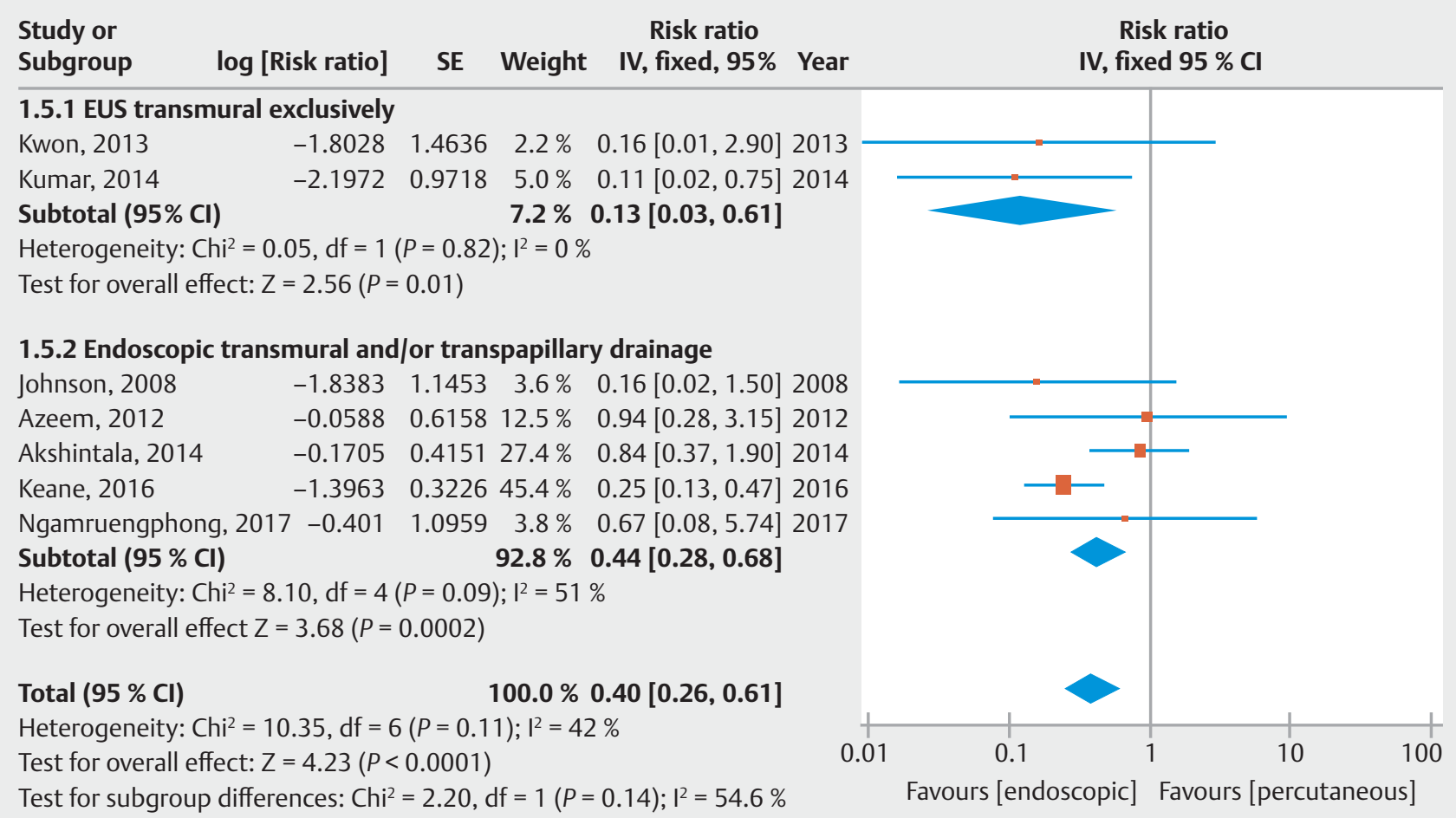

Fig. 2 Forest plot to compare clinical success.

\begin{tabular}{|c|c|c|c|c|}
\hline $\begin{array}{l}\text { Study or } \\
\text { Subgroup }\end{array}$ & Risk ratio] & SE & Weight & $\begin{array}{c}\text { Risk ratio } \\
\text { IV, fixed, } 95 \%\end{array}$ \\
\hline Johnson, 2008 & 0.47 & 1.495 & $13.3 \%$ & $1.60[0.09,29.97]$ \\
\hline Azeem, 2012 & -0.8557 & 1.5192 & $12.9 \%$ & $0.42[0.02,8.38]$ \\
\hline Kwon, 2013 & 0.1484 & 2.0368 & $7.2 \%$ & $1.16[0.02,62.83]$ \\
\hline Akshintala, 2014 & 1.3616 & 1.0957 & $24.7 \%$ & $3.90[0.46,33.42]$ \\
\hline Keane, 2016 & 1.5132 & 1.041 & $27.4 \%$ & $4.54[0.59,34.94]$ \\
\hline Ngamruengphong, 2017 & -2.1088 & 1.4262 & $14.6 \%$ & $0.12[0.01,1.99]$ \\
\hline \multicolumn{2}{|l|}{ Total $(95 \% \mathrm{Cl})$} & \multicolumn{3}{|c|}{$100.0 \% 1.50[0.52,4.37]$} \\
\hline \multicolumn{5}{|c|}{ Heterogeneity: $C h i^{2}=5.71, \mathrm{df}=5(P=0.34) ; I^{2}=12 \%$} \\
\hline \multicolumn{5}{|c|}{ Test for overall effect: $Z=0.75(P=0.46)$} \\
\hline
\end{tabular}

- Fig. 3 Forest plot to compare technical success. the need for a comprehensive systematic review and meta-analysis. This systematic review of observational studies suggests that endoscopic drainage of PFCs should be the preferred option for management compared to percutaneous drainage because it was associated with better clinical success, a lower requirement for re-interventions, and a shorter length of stay in hospital.

When comparing the efficacy of endoscopic with percutaneous drainage for management of PFCs, we found no difference in technical success with no heterogeneity across studies. However, despite similar technical success, clinical success was found to be significantly higher in the endoscopic drainage group. Our initial analysis had moderate heterogeneity which was expected as we had included various types of PFCs. We were able to explore this heterogeneity with a sensitivity analysis after excluding one study [10] evaluating both procedures in the management of WON exclusively. Our results of better clinical success with endoscopic drainage remained consistent even after this sensitivity analysis and may have clinical implications for the management of PFCs. Furthermore, endoscopic creation of a cyst-gastrostomy or cyst-duodenostomy reintroduces pancreatic fluid back into the gastrointestinal tract and 


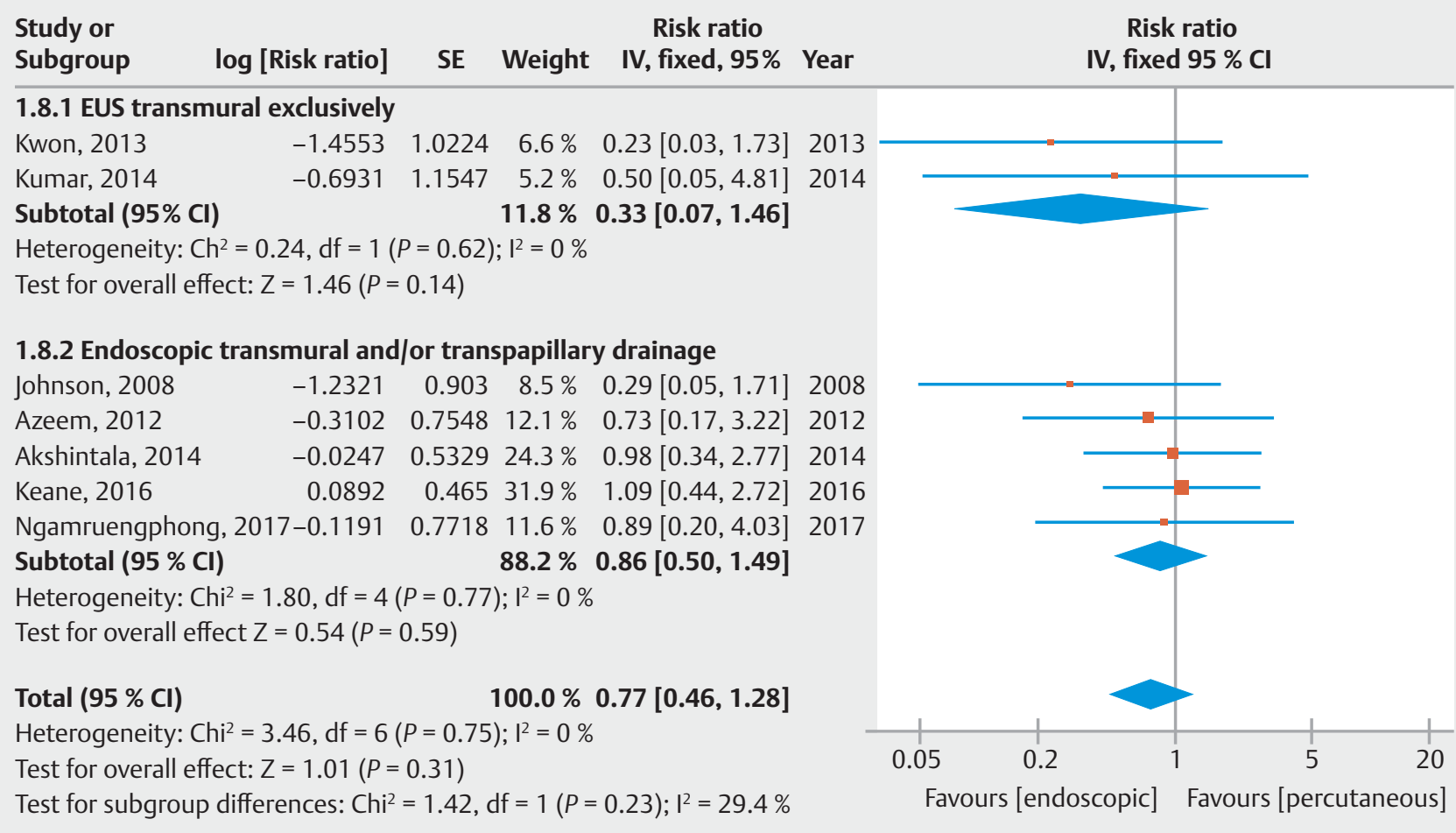

Fig. 4 Forest plot to compare adverse events.

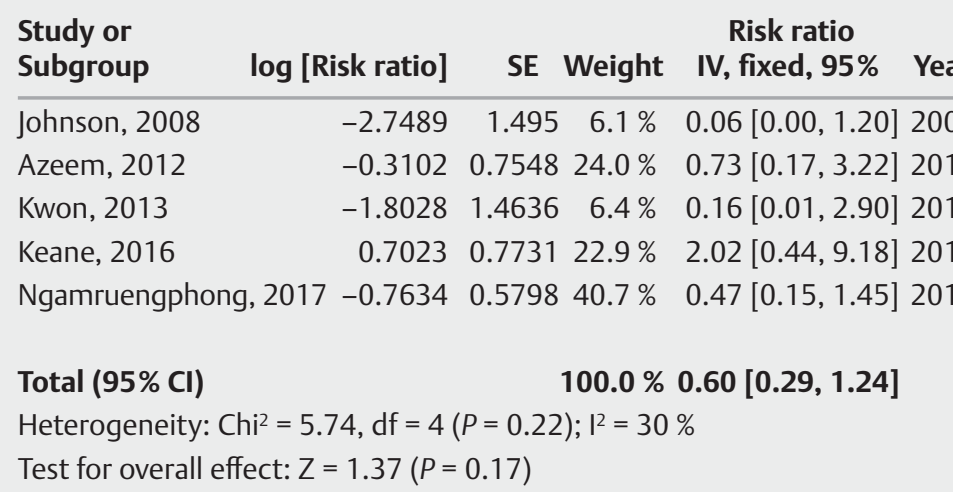

Fig. 5 Forest plot to compare rate of recurrence. is more physiological compared with pancreatic fluid losses with percutaneous drainage. Finally, percutaneous drainage has an external catheter which compromises patients' quality of life, requiring daily care and maintenance and which may result in localized skin irritation.

WON deserves special consideration as such patients have necrosis of pancreatic parenchyma leading to debris in the fluid collection and may not be easily managed by a single drain. Kumar et al. [10] found only $25 \%$ clinical success in the percutaneous drainage group while managing WON. Endoscopic transmural drainage has the advantage in such a scenario because it provides the endoscopist with the ability to perform DEN with mechanical removal of necrotic debris, which cannot be done with small bore percutaneous drains. Although not used by any of the studies included in this meta-analysis, LAMS have a reported clinical success rate of $90 \%$ for management of WON [27].

We did not find any significant difference in post procedure adverse events and rate of recurrence of PFCs between ED and PD. Amongst individual adverse events, we found the rate of bleeding to be higher in the endoscopic group (4\%) compared to the percutaneous group (1.5\%), but this difference was not statistically significant $(P=0.1)$. Bleeding is encountered with endoscopic transmural drainage when there is intervening vas- 


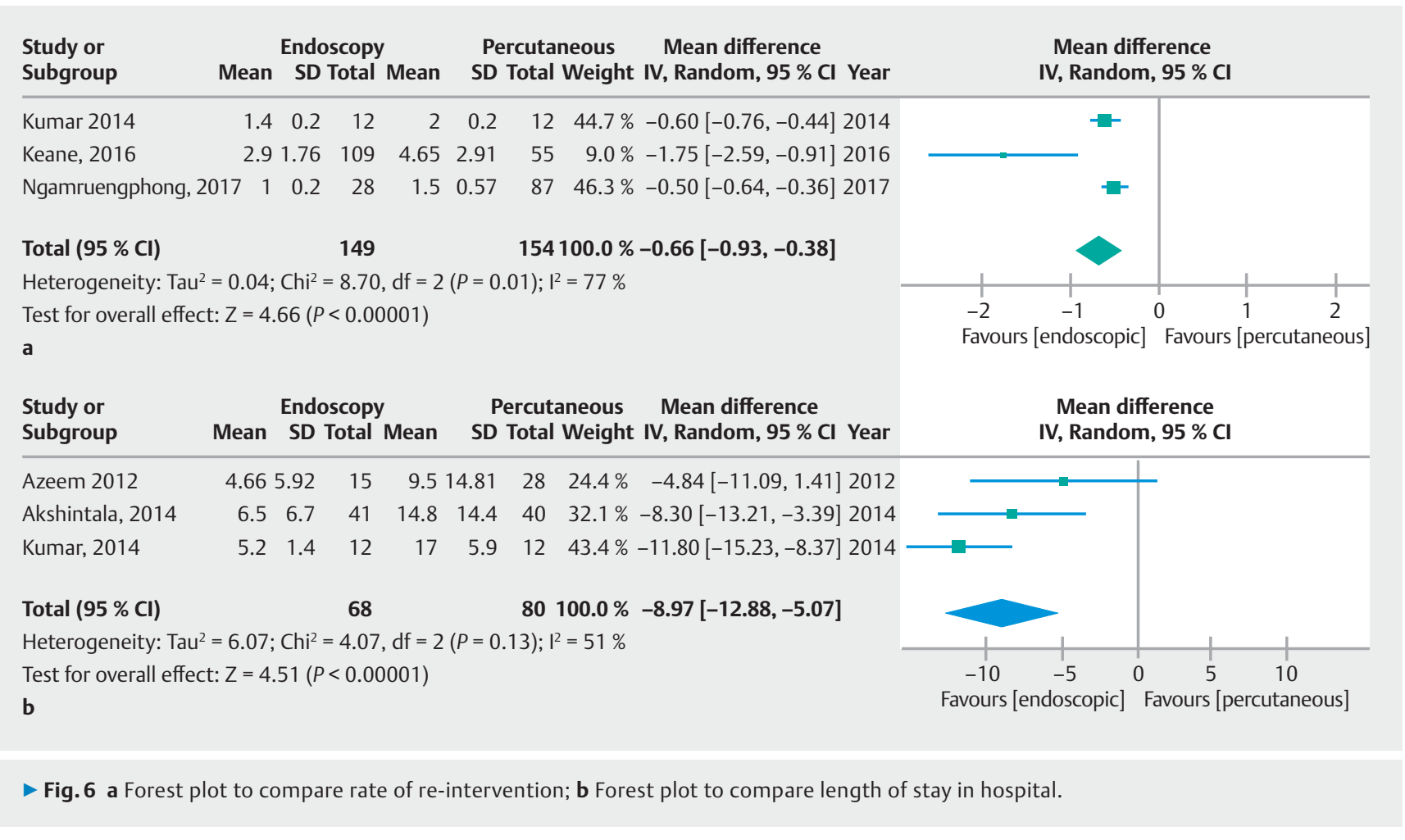

culature at the site of cyst-gastrostomy. However, this complication may decrease with the increasing use of EUS which allows high resolution, real time imaging of the fluid collection and surrounding vasculature thereby allowing precise and safe cyst-gastrostomy or cyst-duodenostomy creation. In this systematic review, only two $[10,22]$ out of seven studies used EUS exclusively. In this analysis, five patients died in the percutaneous group compared to zero in the endoscopic group, but this difference was not statistically significant. However, of note, the studies included in this meta-analysis are retrospective and only two studies $[10,11]$ adjusted for comorbidities in both groups.

The rate of re-intervention was found to be higher in the percutaneous group compared to the endoscopic group. This may be due to problems related to percutaneous drain positions and patency. PFCs tend to collapse with drainage of fluid which could cause drain dislodgment or displacement requiring repositioning. Furthermore, these are smaller caliber drains which tend to occlude necessitating a repeat procedure for drain exchange or upsizing. And lastly, percutaneous drains need to be flushed every day for successful drainage. Patients who are poorly compliant with such protocols are at increased risk of drain malfunction, dislodgment, and infection. The length of hospital stay was also found to be higher in the percutaneous group, which may be partially due to the increased rates of reintervention and lower rates of clinical success. Although we could not perform a formal cost-effective analysis, as such data were not uniformly provided, the higher rates of re-interventions and longer hospital stays together may translate into higher costs associated with percutaneous drainage. Only one study [10] compared the costs and found that the endoscopic option was more cost effective.

This is the first meta-analysis to compare the efficacy and safety of endoscopic versus percutaneous drainage for the management of PFCs. We used a comprehensive search strategy and included all relevant studies. Our analyses may be weakened by inherent limitations of meta-analyses and of the included studies. All of the studies involved were observational studies. Our analysis of clinical success was initially limited by moderate heterogeneity, but with sensitivity analysis we were able to explain heterogeneity by excluding the study evaluating WON exclusively. Our results for clinical success remained consistent after such analysis. Considerable heterogeneity was also found in rates of re-intervention analysis, but due to limited data, this could not be well explored. However, all three studies showed consistently lower rates in the endoscopic group which is reassuring. We were unable to evaluate whether use of EUS resulted in a lower adverse events rate, or if type of stent influenced outcomes as such data were not provided.

\section{Conclusions}

This meta-analysis confirms that endoscopic drainage should be the preferred therapeutic modality for symptomatic PFCs compared to percutaneous drainage, as it is associated with significantly better clinical success, lower re-intervention rates, and shorter hospital length of stay. Additionally, with recent improvements in endoscopic techniques not included in this analysis, specifically the routine use of EUS and LAMS, these discrepancies could be expected to be even more pronounced than demonstrated in our study. Further study is needed. 
Competing interests

Michel Kahaleh MD has received grant support from Boston Scientific, Fujinon, EMcison, Xlumena Inc., W. L. Gore, MaunaKea, Apollo Endosurgery, Cook Endoscopy, ASPIRE Bariatrics, GI Dynamics, NinePoint Medical, Merit Medical, Olympus and MI Tech. He is a consultant for Boston Scientific, Xlumena Inc., Concordia Laboratories Inc, ABBvie, and MaunaKea Tech. All other authors have no conflicts of interest to report.

\section{References}

[1] Peery AF, Dellon ES, Lund J et al. Burden of gastrointestinal disease in the United States: 2012 update. Gastroenterology 2012; 143: 11791187.e3

[2] Poornachandra KS, Bhasin DK, Nagi B et al. Clinical, biochemical, and radiologic parameters at admission predicting formation of a pseudocyst in acute pancreatitis. J Clin Gastroenterol 2011; 45: 159-163

[3] Forsmark CE, Bhasin DK, Nagi B et al. AGA Institute technical review on acute pancreatitis. Gastroenterology 2007; 132: 2022-2044

[4] Sheu Y, Furlan A, Almusa O et al. The revised Atlanta classification for acute pancreatitis: a CT imaging guide for radiologists. Emerg Radiol 2012; 19: 237-243

[5] Kleeff J, Diener MK, Z'graggen K et al. Distal pancreatectomy: risk factors for surgical failure in 302 consecutive cases. Ann Surg 2007; 245: $573-582$

[6] Kooby DA, Gillespie T, Bentrem D et al. Left-sided pancreatectomy: a multicenter comparison of laparoscopic and open approaches. Ann Surg 2008; 248: $438-446$

[7] Goh BK, Tan YM, Chung YF et al. Critical appraisal of 232 consecutive distal pancreatectomies with emphasis on risk factors, outcome, and management of the postoperative pancreatic fistula: a 21-year experience at a single institution. Arch Surg 2008; 143: 956 - 965

[8] van Santvoort HC, Besselink MG, Bakker O] et al. A step-up approach or open necrosectomy for necrotizing pancreatitis. NEJM 2010; 362 : $1491-1502$

[9] Bakker OJ, van Santvoort HC, van Brunschot S et al. Endoscopic transgastric vs surgical necrosectomy for infected necrotizing pancreatitis: a randomized trial. JAMA 2012; 307: 1053 - 1061

[10] Kumar N, Conwell DL, Thompson CC. Direct endoscopic necrosectomy versus step-up approach for walled-off pancreatic necrosis: comparison of clinical outcome and health care utilization. Pancreas 2014; 43: $1334-1339$

[11] Akshintala VS, Saxena P, Zaheer A et al. A comparative evaluation of outcomes of endoscopic versus percutaneous drainage for symptomatic pancreatic pseudocysts. Gastrointest Endosc 2014; 79: 921 928 ; quiz 983 e2, 983 e 5

[12] Hookey LC, Debroux S, Delhaye M et al. Endoscopic drainage of pancreatic-fluid collections in 116 patients: a comparison of etiologies, drainage techniques, and outcomes. Gastrointest Endosc 2006; 63: $635-643$
[13] Baron TH, Thaggard WG, Morgan DE et al. Endoscopic therapy for organized pancreatic necrosis. Gastroenterology 111755-764

[14] Hammad TA, Khan MA, Alastal Y et al. Efficacy and safety of lumenapposing metal stents in EUS guided transmural drainage of pancreatic fluid collections: a systematic review and meta-analysis. Am J Gastroenterol 2016; 111: 16

[15] Mukai S, Itoi T, Baron TH et al. Endoscopic ultrasound-guided placement of plastic vs. biflanged metal stents for therapy of walled-off necrosis: a retrospective single-center series. Endoscopy 2015; 47: $47-55$

[16] Liberati A, Altman DG, Tetzlaff J et al. The PRISMA statement for reporting systematic reviews and meta-analyses of studies that evaluate healthcare interventions: explanation and elaboration. BMJ 2009; 339: b2700

[17] Wells GA, Shea B, O'Connell D et al. The Newcastle-Ottawa scale (NOS) for assessing the quality of nonrandomized studies in metaanalyses. The Ottawa Hospital Research Institute;http://www.ohri.ca/ programs/clinical_epidemiology/oxford.asp

[18] Sweeting MJ, Sutton AJ, Lambert PC. What to add to nothing? Use and avoidance of continuity corrections in meta-analysis of sparse data Stat Med 2004; 23: 1351 - 1375

[19] Friedrich JO, Adhikari NK, Beyene J. Inclusion of zero total event trials in meta-analyses maintains analytic consistency and incorporates all available data. BMC Med Res Methodol 2007; 7: 5

[20] Deeks J], Higgins JPT, Altman DG on behalf of the Cochrane Statistical Methods Group. Chapter 9: Analysing data and undertaking metaanalyses. Cochrane Handbook for Systematic Reviews of Interventions Version 5.0.1 [updated September 2008]. The Cochrane Collaboration 2008; 2008

[21] Azeem N, Baron TH, Topazian MD et al. Outcomes of endoscopic and percutaneous drainage of pancreatic fluid collections arising after pancreatic tail resection. J Am Coll Surg 2012; 215: 177-185

[22] Kwon YM, Gerdes H, Schattner MA et al. Management of peripancreatic fluid collections following partial pancreatectomy: a comparison of percutaneous versus EUS-guided drainage. Surg Endosc 2013; 27: $2422-2427$

[23] Keane MG, Sze SF, Cieplik N et al. Endoscopic versus percutaneous drainage of symptomatic pancreatic fluid collections: a 14-year experience from a tertiary hepatobiliary centre. Surg Endosc 2016; 30: $3730-3740$

[24] Johnson MD, Walsh RM, Henderson JM et al. Surgical versus nonsurgical management of pancreatic pseudocysts. J Clin Gastroenterol 2009; 43: $586-590$

[25] Ngamruengphong S, Fargahi F, Kamal A et al. Comparative evaluation of endoscopic drainage vs percutaneous drainage for pancreatic leak following distal pancreatectomy. Gastrointest Endosc 2017; 85: AB217

[26] Akshintala VS, Zaheer A, Rana U et al. Comparative evaluation of outcomes of endoscopic versus percutaneous drainage for symptomatic pancreatic pseudocysts. Gastrointest Endosc 2012; 75: AB508

[27] Hammad T, Khan MA, Alastal Y et al. Efficacy and safety of lumen-apposing metal stents in management of pancreatic fluid collections: Are they better than plastic stents? A systematic review and metaanalysis Dig Dis Sci 2017: doi:10.1007/s10620-017-4851-0 [Epub ahead of print] 


\section{Appendix 1}

\section{PubMed search strategy}

(()((Pancreatic-fluid- collection* OR pancreatic fluid collection OR PFC OR walled-off- pancreatic-necros* OR WOPN OR pancreatic-abscess* OR peripancreatic-fluid- collection* OR ((Pancreas OR pancreatic OR intrapancreatic OR peripancreatic) AND (pancreatic pseudocyst* OR pseudocyst* OR walled-off- necros* OR WON OR necrotic-collection* OR cystic-collection* OR necrosis[mesh] OR cysts[mesh:no exp])) OR \&quot;pancreatic pseudocyst\&quot;[Mesh] OR necrosectomy OR drain OR drainage $]))))$ AND ((((endoscopic- ultrasonograph* OR endoscopic-ultrasound* OR endosonograph* OR EUS OR interventional- ultrasonograph* OR endoscopic-drainage* OR therapeutic-EUS* OR interventional-EUS* OR interventional-ultrasound* OR Intravascular-Ultrasonograph* OR Echo-Endoscop* eus-guided OR eus guided OR direct endoscopic OR transcutaneous endoscopic OR Ultrasonic-Endoscop* OR \&quot;Ultrasonography, Interventional\&quot;[Mesh] OR \&quot;Endosonography\&quot;[Mesh] OR endoscopy, gastrointestinal[mesh] OR endoscopic ultrasound-guided fine needle aspiration [mesh] AND percutaneous)))) 